\title{
Supernova neutrinos: fast flavor conversions near the core
}

\author{
Manibrata Sen \\ Tata Institute of Fundamental Research, Homi Bhabha Road, Mumbai, 400005, India. \\ manibrata.sen@gmail.com .
}

\begin{abstract}
Neutrino flux streaming from a supernova can undergo rapid flavor conversions almost immediately above the core. Focusing on this region, we study these fast conversions using a linear stability analysis. We find that, for realistic angular distributions of neutrinos, fast conversions can occur within a few nanoseconds in regions just above the neutrinosphere. Our results also show that neutrinos travelling towards the core make fast conversions more rapid. These conversions, if they exist, can have significant implications for supernova explosion mechanism and nucleosynthesis.
\end{abstract}

Keywords: supernova neutrinos, fast flavor conversions

TIFR/TH/17-08

\section{Introduction}

Core collapse supernovae (SN) offer a fascinating environment to study neutrino flavor evolution in dense environments. Very recently, it was predicted that fast flavor conversions, occurring with a rate $\mu \sim \sqrt{2} G_{F} n_{\nu}\left(n_{\nu}\right.$ is the neutrino density), can happen very near the SN core, as opposed to the well-known collective effects, occurring at $r \sim \mathcal{O}\left(10^{2}\right) \mathrm{km}[3,4]$ or the MSW effect at $r \sim \mathcal{O}\left(10^{3}\right) \mathrm{km}$ $[1,2]$. A necessary condition for this seemed to be a non-trivial angular distribution in the neutrino emission spectrum $[5,6]$. Based on these claims, we make a detailed study of these fast flavor conversions. We focus on regions close to the SN core and hence model the source as a flat geometry as shown in the left panel of Fig. 1. We redo the linear stability analysis(LSA) with a physically well motivated angular emission spectrum, where the $\nu_{e}$, which decouple later than the $\bar{\nu}_{e}, \nu_{x}$, have a larger flux and wider angular distribution than the latter. We study possible instabilities for neutrino flavor evolution in space as well as time, and for the first time, include backward going modes also. Finally, we verify our LSA results with numerical results from the fully non-linear evolution.

\section{Set-up of the problem}

Neutrino flavor evolution in a dense media is explained with space-time dependent Wigner functions $\varrho_{\mathbf{p}, \mathbf{x}, t}$ with momentum $\mathbf{p}$ at position $\mathbf{x}$ and time $t$. The equation of motion (EoM) is

$$
\partial_{t} \varrho_{\mathbf{p}, \mathbf{x}, t}+\mathbf{v}_{\mathbf{p}} \cdot \nabla_{\mathbf{x}} \varrho_{\mathbf{p}, \mathbf{x}, t}=-i\left[\Omega_{\mathbf{p}, \mathbf{x}, t}, \varrho_{\mathbf{p}, \mathbf{x}, t}\right],
$$

Here we have neglected external forces acting on the system as well as collisions. The Hamiltonian matrix is $\Omega_{\mathbf{p}}=\Omega_{\mathrm{vac}}+\Omega_{\mathrm{MSW}}+\Omega_{\nu \nu}$, where $\Omega_{\mathrm{vac}}=$ $\operatorname{diag}(-\omega / 2,+\omega / 2)$ is the vacuum term with $\omega=\Delta m^{2} / 2 E ; \Omega_{\mathrm{MSW}}=\lambda \operatorname{diag}(1,0)$ is the matter term with $\lambda=\sqrt{2} G_{F} n_{e}$, where $n_{e}$ is the electron density; and $\Omega_{\nu \nu}=\sqrt{2} G_{F} \int \frac{d^{3} \mathbf{q}}{(2 \pi)^{3}}\left(\varrho_{\mathbf{q}}-\bar{\varrho}_{\mathbf{q}}\right)\left(1-\mathbf{v}_{\mathbf{p}} \cdot \mathbf{v}_{\mathbf{q}}\right)$ is the multi-angle neutrino-neutrino 

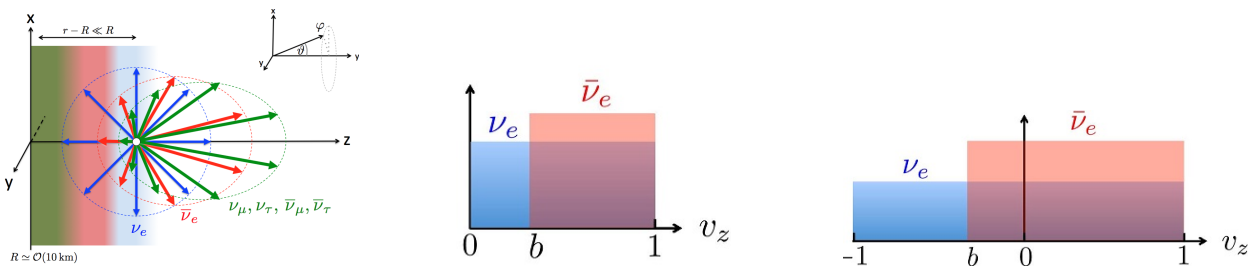

Fig. 1. Left panel shows schematic polar plots of the angular distributions of the $\nu_{e}$ (blue), $\bar{\nu}_{e}$ (red), and $\nu_{x}$ (green) emission fluxes. Middle panel shows a spectrum with no ingoing $\nu_{e}$ or $\bar{\nu}_{e}$, while the right panel shows ingoing $\nu_{e}$ and $\bar{\nu}_{e}$.

interaction term. Since the total number of neutrinos is always conserved, we can write

$$
\varrho_{\omega, v_{z}, \varphi}=\frac{1}{2} \operatorname{Tr}\left(\varrho_{\omega, v_{z}, \varphi}\right) \mathbb{I}+\Phi_{\nu} \frac{g_{\omega, v_{z}, \varphi}}{2}\left(\begin{array}{cc}
s_{\omega, v_{z}, \varphi} & S_{\omega, v_{z}, \varphi} \\
S_{\omega, v_{z}, \varphi}^{*} & -s_{\omega, v_{z}, \varphi}
\end{array}\right)
$$

and drop the trace term. Here $S_{\omega, v_{z}, \varphi} \ll 1$ and $s_{\omega, v_{z}, \varphi}^{2}+\left|S_{\omega, v_{z}, \varphi}\right|^{2}=1$. Also $\Phi_{\nu}$ is the normalization of the differential spectrum $g_{\omega, v_{z}, \varphi}$, chosen accordingly. As neutrinos are produced as flavor eigenstates, no oscillation occurs as long as $S_{\omega, v_{z}, \varphi}=0$. We linearize the equations in this small parameter $S_{\omega, v_{z}, \varphi}$ to get an eigenvalue equation $[7]$

$$
\begin{aligned}
i\left(\partial_{t}+v_{z} \partial_{z}+\mathbf{v}_{T} \cdot \partial_{T}\right) S_{\omega, v_{z}, \varphi}= & {\left[\omega+\lambda+\mu \int d \Gamma^{\prime}\left(1-v_{z} v_{z}^{\prime}-\mathbf{v}_{T} \cdot \mathbf{v}_{T}^{\prime}\right) g_{\omega^{\prime}, v_{z}^{\prime}, \varphi^{\prime}}\right] S_{\omega, v_{z}, \varphi} } \\
& -\mu \int d \Gamma^{\prime}\left(1-v_{z} v_{z}^{\prime}-\mathbf{v}_{T} \cdot \mathbf{v}_{T}^{\prime}\right) g_{\omega^{\prime}, v_{z}^{\prime}, \varphi^{\prime}} S_{\omega^{\prime}, v_{z}^{\prime}, \varphi^{\prime}},
\end{aligned}
$$

where $\mathbf{v}_{T}$ is the velocity vector of the neutrino projected on the $x-y$-plane. The important quantity here is the difference in the diiferential spectrum, given by $g_{\omega, v_{z}, \varphi} \propto d \phi_{\nu_{e}} / d \Gamma-d \phi_{\nu_{x}} / d \Gamma$ for neutrinos and $\propto d \phi_{\nu_{x}} / d \Gamma-d \phi_{\bar{\nu}_{e}} / d \Gamma$ for antineutrinos. Keeping in mind that the angular spectrum of emission should be different for neutrinos and antineutrinos, we choose the following schematic spec$\operatorname{trum} g_{\omega, v_{z}, \varphi}=\frac{1}{2 \pi}\left[(1+a) \delta(\omega) \Theta\left(v_{z}\right) \Theta\left(1-v_{z}\right)-\delta(\omega) \frac{1}{(1-b)} \Theta\left(v_{z}-b\right) \Theta\left(1-v_{z}\right)\right]$ as shown in Fig. 1 (middle panel). Here $a$ denotes the neutrino-antineutrino asymmetry whereas $b$ controls the difference in zenith angle distribution. Such a "non-trivial" angular emission spectrum seems to be crucial for fast conversion.

To solve the eigenvalue equation, we take $S=Q e^{-i\left(\Omega_{t} t+\Omega_{z} z\right)}$, where $\Omega_{(t, z)}=$ $\gamma_{(t, z)}+i \kappa_{(t, z)}$ can take complex values. A non-zero positive $\kappa_{(t, z)}$ causes an exponential growth in $S$, thereby signalling an instability. It is important to mention that since we are looking for fast conversions, we can integrate out $\omega$ from the spectrum and effectively set $\omega / \mu \rightarrow 0$.

\section{Results}

Armed with this formalism, we look for instabilities for evolution in time $\left(\Omega_{z} \rightarrow\right.$ $0)$ and in space $\left(\Omega_{t} \rightarrow 0\right)$. We show a contour plot $\kappa_{z}$ for different values of $a$ and $b$ in Fig. 2. A common feature of all these plots is that no fast conversion takes place if $b=0$, thereby indicating that a non-trivial spectrum might be necessary for fast conversions. Also, matter suppresses these growths.

Similarly, in Fig. 3, we plot the growth rates for $\kappa_{t}$. We also show similar instabilities for a spectrum including backward travelling modes as $g_{\omega, v_{z}, \varphi}=$ $\frac{1}{2 \pi}\left[\frac{1+a}{2} \delta(\omega) \Theta\left(1+v_{z}\right) \Theta\left(1-v_{z}\right)-\delta(\omega) \frac{1}{(1-b)} \Theta\left(v_{z}-b\right) \Theta\left(1-v_{z}\right)\right]$ as shown in Fig. 1 

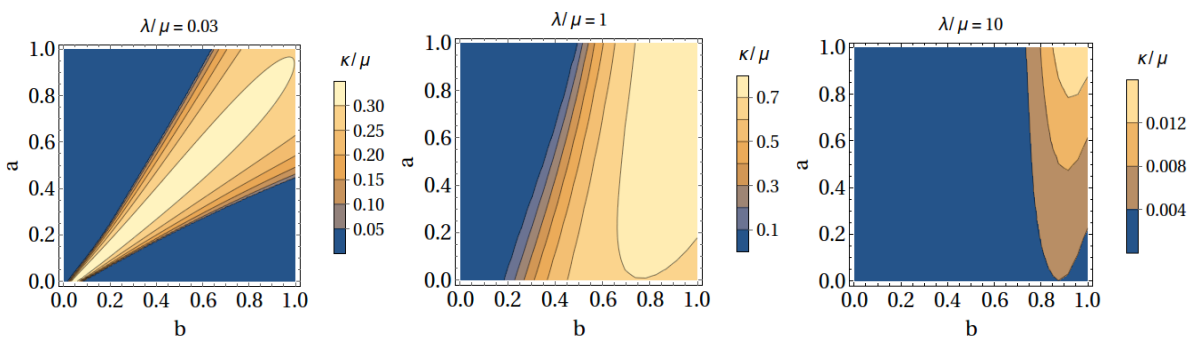

Fig. 2. Instability growth rates for evolution in space for three different values of $\lambda / \mu=$ $0.03,1$, and 10 , respectively from left to right.
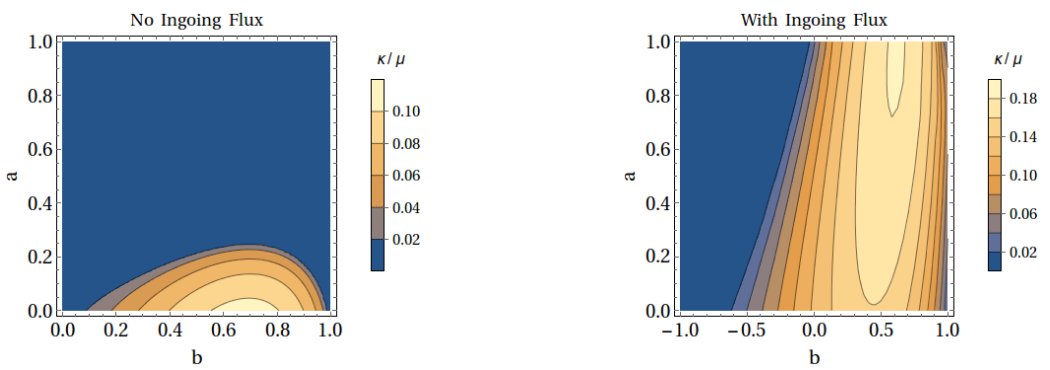

Fig. 3. Instability growth rates for evolution in time. Left: without inward going modes. Right: with inward going modes.
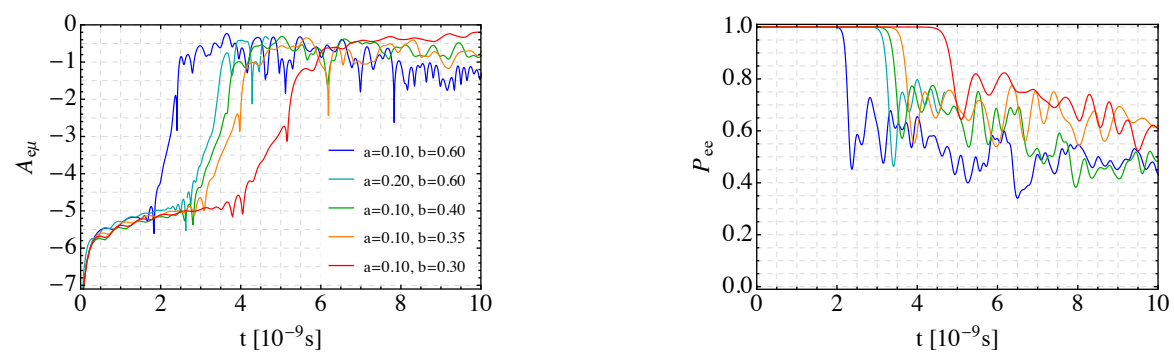

Fig. 4. Instability growth for evolution in time. Left panel shows the quantity $A_{e \mu}=$ $\log _{10}|S|$ giving the extent of flavor conversion. Right panel shows the electron neutrino survival probabilities $P_{e e}$ for same values of $a$ and $b$.

(right panel). We note that inclusion of backward going modes increases fast conversions. Interestingly, matter suppression does not occur for time evolution, as suggested in [9]. To verify our results from LSA, we have also solved the fully non-linear EoMs for specific values of $a$ and $b$. The results shown in Fig. 4 indicate that indeed complete flavor averaging takes place within a few nanoseconds. One is also led to speculate whether it is important to have a crossing in the angular spectrum for development of fast conversions, as is evident from all the above cases. Further details are worked out in [8].

\section{Conclusion}

Thus we notice that for a non-trivial distribution of angular spectrum, we can indeed get rapid flavor turn-overs in time, even if in space the growth is suppressed by matter effects. Also, backward travelling modes near the neutrinosphere aid in fast conversion. If these fast conversions indeed take place so near the SN core, they may have important implications for supernova explosion mechanism and nucleosynthesis. 


\section{References}

1. L. Wolfenstein, Phys. Rev. D 17, 2369 (1978). doi:10.1103/PhysRevD.17.2369

2. S. P. Mikheev and A. Y. Smirnov, Sov. J. Nucl. Phys. 42, 913 (1985) [Yad. Fiz. 42, 1441 (1985)].

3. H. Duan, G. M. Fuller, J. Carlson and Y. Z. Qian, Phys. Rev. D 74, 105014 (2006) doi:10.1103/PhysRevD.74.105014 [astro-ph/0606616].

4. S. Hannestad, G. G. Raffelt, G. Sigl and Y. Y. Y. Wong, Phys. Rev. D 74, 105010 (2006) Erratum: [Phys. Rev. D 76, 029901 (2007)] doi:10.1103/PhysRevD.74.105010, 10.1103/PhysRevD.76.029901 [astro$\mathrm{ph} / 0608695]$.

5. R. F. Sawyer, Phys. Rev. Lett. 116, no. 8, 081101 (2016) doi:10.1103/PhysRevLett.116.081101 [arXiv:1509.03323 [astro-ph.HE]].

6. S. Chakraborty, R. S. Hansen, I. Izaguirre and G. Raffelt, JCAP 1603, no. 03, 042 (2016) doi:10.1088/1475-7516/2016/03/042 [arXiv:1602.00698 [hep-ph]].

7. A. Banerjee, A. Dighe and G. Raffelt, Phys. Rev. D 84 (2011) 053013 doi:10.1103/PhysRevD.84.053013 [arXiv:1107.2308 [hep-ph]].

8. B. Dasgupta, A. Mirizzi and M. Sen, arXiv:1609.00528 [hep-ph].

9. B. Dasgupta and A. Mirizzi, Phys. Rev. D 92 (2015) no.12, 125030 doi:10.1103/PhysRevD.92.125030 [arXiv:1509.03171 [hep-ph]]. 